interaction) and in the folkdance intervention (IRR $=1.68$, 95\% CI:1.03 -2.73). Social dancing did not prevent falls or its associated risk factors among villages' residents. Modified dance programmes that contain "training elements" to better approximate structured exercise programs, targeted at low and high-risk participants, warrant investigation.

\section{DOES DEPRESCRIBING FALL-ASSOCIATED DRUGS REDUCE FALLS AND ITS COMPLICATIONS?: A SYSTEMATIC REVIEW}

J. Lee ${ }^{2,4,6}$, A. Negm ${ }^{1,2}$, E. Wong 5 , A. Holbrook ${ }^{3,6,7}, 1$. McMaster University, Hamilton, Ontario, Canada, 2. Geriatric Education and Research in Aging Sciences (GERAS) Centre, Hamilton, Ontario, Canada, 3. Center for Evaluation of Medicines, Hamilton, Ontario, Canada, 4. Division of Geriatric Medicine, McMaster University, Hamilton, Ontario, Canada, 5. Department of Medicine, McMaster University, Hamilton, Ontario, Canada, 6. Department of Clinical Epidemiology \& Biostatistics, McMaster University, Hamilton, Ontario, Canada, 7. Division of Clinical Pharmacology \& Toxicology, McMaster University, Hamilton, Ontario, Canada

Falls are the leading cause of injury and injury-related hospitalizations for seniors in Canada with annual healthcare costs exceeding $\$ 2$ billion. Despite limited evidence of effectiveness, the withdrawal (discontinuation or dose reduction) of "fall-risk increasing drugs" (FRIDs) is typically part of falls prevention strategies and hospital accreditation initiatives. The study objectives were to determine the preventative efficacy of FRID withdrawal on falls and fall-related complications. An electronic search was conducted in MEDLINE, EMBASE, CENTRAL and CINAHL. A grey literature search included trial registries and conference abstracts. All randomized controlled trials in adults age $\geq 65$ evaluating FRID withdrawal compared to usual care on falls rate or incidence, fall-related injuries, fractures or hospitalizations and/or adverse effects related to the intervention were included. Two reviewers independently screened eligible studies, abstracted data and assessed risk of bias. The GRADE criteria were used to rate overall confidence in effect estimates for outcomes. Five trials involving 1309 participants met eligibility criteria for inclusion. A FRID withdrawal strategy did not significantly change the rate of falls ( RaR $0.98,95 \%$ CI 0.63 to 1.51), number of fallers (RR $1.06,95 \%$ CI 0.84 to 1.34 ) or rate of fall-related injuries (RaR 0.89 , 95\% CI 0.57 to 1.39 ) over a 6 to 12 month follow-up period. There is insufficient evidence that a FRID withdrawal strategy is effective for preventing falls. Based on very low quality evidence, it is uncertain whether FRID withdrawal leads to any appreciable clinically important benefit. Data evaluating the potential harms of FRID withdrawal is lacking.

\section{EXERCISE TO PREVENT FALLS IN OLDER ADULTS: AN UPDATED SYSTEMATIC REVIEW AND META-ANALYSIS}

C. Sherrington ${ }^{1}$, Z. Michaleff ${ }^{1,2}$, N. Fairhall ${ }^{1}$,

A. Tiedemann ${ }^{1}$, J. Whitney ${ }^{3}$, R. Cumming ${ }^{1}$, J. Close $^{4}$, S. Lord ${ }^{4}, 1$. University of Sydney, Sydney, New South Wales, Australia, 2. Keele University, Keele, United Kingdom, 3. King's College Hospital, London, United Kingdom, 4.
University of New South Wales, Sydney, New South Wales, Australia

Previous meta-analyses have found exercise as a single intervention prevents falls in older people. This updated systematic review with random effects meta-analysis and metaregression aimed to test whether this effect is still present when new trials are added and explore trial characteristics associated with greater fall prevention effects. One hundred comparisons from 89 randomised trials with 19,869 participants were available for meta-analysis. Overall, exercise reduced the rate of falls in community dwelling older people by $21 \%$ (pooled rate ratio $0.79,95 \%$ CI 0.73 to 0.85 , $\mathrm{p}<0.001, \mathrm{I}^{2} 47 \%, 67$ comparisons) with greater effects seen from exercise programs that challenged balance and involved more than three hours per week of exercise. Together these variables explained $76 \%$ of the between-trial heterogeneity and in combination led to a $39 \%$ reduction in falls (IRR $0.61,95 \%$ CI 0.53 to $0.72, \mathrm{p}<0.001)$. Exercise also had a fall prevention effect in community-dwelling people with Parkinson's disease (pooled rate ratio $0.47,95 \%$ CI 0.30 to $0.73, \mathrm{p}=0.001, \mathrm{I}^{2} 65 \%$, 6 comparisons) or cognitive impairment (pooled rate ratio $0.55,95 \%$ CI 0.37 to $0.83, p=0.004$, $\mathrm{I}^{2} 21 \%, 3$ comparisons) but not in residential care settings or among stroke survivors, people with severe visual impairment or people recently discharged from hospital. Exercise as a single intervention can prevent falls in community dwelling older people, promising results are seen in people with Parkinson's disease or cognitive impairment but its impact in other clinical groups and aged care facility residents requires further investigation.

\section{FALLS AND THE SOCIAL ISOLATION OF OLDER ADULTS IN THE NATIONAL HEALTH AND AGING TRENDS STUDY}

J. Pohl, B.B. Cochrane, K.G. Schepp, N.F. Woods, University of Washington School of Nursing, Seattle, Washington

Falls among community-dwelling older adults can be life threatening. While an association between social isolation and falls has been described, the nature of that relationship is not well documented and could be important for fall prevention interventions. Study objectives were to describe the incidence of falls, prevalence of social isolation, and extent to which social isolation predicts falls in older adults. Secondary analysis of longitudinal data from the National Health and Aging Trends Study, involving a nationally representative sample of Medicare beneficiaries, included four rounds of annual interviews in participants' homes (round one $n=7,609$ ). Social isolation was operationalized for the current analysis as a multiple-indicator, domain-inclusive construct based upon the Social Network Index. Falling during the previous year was self-reported. Incidence of falls ranged from $22.4-26.2 \%$ across the four rounds. Social isolation prevalence ranged from $19.8-21.9 \%$. The probability of falling increased with each increase in social isolation construct score. Even after adjusting for age, gender, and education, social isolation significantly predicted falling $(O R=1.08 ; C I=1.02-1.14)$. Adding self-reported general health, depression, and worry about falling to the model weakened the relationship between social isolation and falls $(O R=1.02 ; C I=0.96-1.08)$. Adding the Short Physical Performance Battery, assistive mobility device use, and 
activities of daily living to the model weakened the relationship further $(O R=0.99 ; C I=0.94-1.04)$. Social isolation as a predictor of falls is partially explained by the strong relationship between social isolation and physical performance. Fall-prevention interventions targeting social isolation could have an important impact on physical performance and future falls.

\section{SESSION 635 (PAPER)}

\section{CANCER-RELATED MORBIDITY AND MORTALITY ISSUES}

\section{A CLINICAL SCORE TO PREDICT THE EARLY DEATH AT 100 DAYS IN ELDERLY METASTATIC CANCERS}

R. Boulahssass, S. Gonfrier, M. Sanchez, C. Rambaud, A. Gary, J. Turpin, I. Bereder, O. Guerin, CHU de Nice UCOG PACA East, Nice, France

Background: Trying to predict the very early death after a CGA is difficult in elderly metastatic cancers. Last year, we presented a clinical score to predict this risk in 815 elderly cancer patients (Boulahssass et al 9511 ASCO 2015). The aim of this new study is the next step by developing a score to estimate the risk of early death at 100 days in metastatic cancers $(\mathrm{MC})$ in order to have the collective wisdom not to overtreat this population.100 days is nearly 3 months, if patients are going to die within 3 months, it's maybe necessary to provide them best supportive care alone.

Methods: This is a multicentric and prospective cohort study approved by an ethics committee. At the baseline, a standardized CGA was performed (MMSE,MNA,Grip strength, ADL, IADL,CIRSg, Charlson, lee,PS,Gait speed, QLQc30, G8, Balducci), type and localization of metastases were collected. During the follow up of 100 days, events, treatments made and targeted geriatric interventions were collected. A multivariate logistic regression permits to select risk factors. The internal validation was performed by a bootstrap with randomized samples. Score points were assigned to each risk factors by using the $\beta$ coefficient. The accuracy of the score was assessed with the mean c-statistic and the calibration with the Hosmer-Lemeshow goodness of fit test.

Results: In the cohort 312 patients had a MC with a median age of $82 \mathrm{y}$. The indepedent predictors of death at 100 days in MC were: Age > 85y (OR 2,1 p=0,03), Metastatic localizations (ML): 2ML (OR 2,4 p=0,004), >2 ML (OR 6,3 p=0,001),MNA $<17$ (OR 8,7 p<0,0001) or $\leq 23,5$ and $\geq 17$ (OR 5,4 p=0,002), Home confinement (OR $1,8 \mathrm{p}=0,047), \mathrm{ADL}<5,5$ (OR 2,1 $\mathrm{p}=0,017$ ), Cancers with global risk of early death at 100 days $>30 \%$ (OR 2,05 $\mathrm{p}=0,016$ )

We assigned in the score: 3 points for: $\mathrm{MNA} \leq 23,5, \mathrm{ML}>2$ and 1 point for home confinement, $\mathrm{ADL}<5,5, \mathrm{ML}=2$, age $>85 y$ and types of cancers at risk $>30 \%$

The risk of death at 100 days in MC was $4 \%$ for 0 to 2 pts, $18 \%$ for 3 to 4 pts, $33 \%$ for 5 pts and $44 \%$ for 6 pts and $83 \%$ for $>6$ pts.

Conclusion: In daily practice, this score should help to avoid unnecessary treatment for patients with a high risk of death, especially for those with a score $>6$.

\section{COGNITIVE FUNCTION AND HORMONAL THERAPY ADHERENCE IN A COHORT OF OLDER SURVIVORS: CALGB 369901}

S. Bluethmann ${ }^{4}$, C. Alfano ${ }^{2}$, J. Clapp ${ }^{1}$, L. Faul ${ }^{1}$, G. Luta ${ }^{1}$, J. Mandelblatt ${ }^{1}, 1$. Georgetown University, Washington, District of Columbia, 2. American Cancer Society, Washington, District of Columbia, 4. Pennsylvania State University, Hershey, Pennsylvania

Background: Adjuvant hormonal therapy is the standard of care for estrogen-receptor-positive $(\mathrm{ER}+)$ breast cancer survivors, but up to half of survivors do not adhere to recommended treatment ( 5 years + ). Adherence may be more complicated in women older than 65 years (the majority of survivors) due to adverse effects of hormonal therapy, such as cognitive impairment. However, the relationship between cognitive function and hormonal therapy adherence is poorly understood. The objective of this study is to examine the relationship of cognitive function and hormonal therapy adherence over 5 years in older breast cancer survivors.

Methods: A cohort of cognitively intact ER+, non-metastatic breast cancer survivors (65 years and older) that initiated hormonal therapy were recruited from 78 sites between 2004-2011 ( $\mathrm{n}=909$ ). Follow-up data were collected at 6 months and annually for up to 7 years. Cognitive function was self-reported using EORTC-QLC30. Group-based trajectory modeling determined groups based on the highest probability of membership and logistic regression was used to test associations with discontinuation of hormonal therapy before at least 5 years of prescribed medication use.

Results: The mean cognitive score for survivors at baseline was 93.2 points $(\mathrm{SD}=12.3)$. Most breast cancer survivors were in early to middle stages of disease (i.e., stage $1=47.4 \%$, stage $2 \mathrm{a}=31.4 \%$ and stage $2 \mathrm{~b}-3=21.3 \%$ ). Approximately $30 \%$ of women reported early discontinuation of hormonal therapy. Many women $(42 \%)$ had high cognitive function that remained stable over time; more $(49.1 \%)$ showed a phase shift (i.e., slight decline) in cognitive function with time. Only $9.2 \%$ of survivors showed a pattern of accelerated cognitive decline over time. Survivors with accelerated decline were more likely than those who maintained high function to discontinue hormonal therapy (OR 1.49: 95\% CI, 0.88-2.55, $\mathrm{p}=0.14$ ), controlling for age, stage and prior use of chemotherapy. Additionally, survivors who exhibited a phase shift in cognitive function were significantly more likely than survivors who maintained high function to discontinue hormonal therapy (OR 1.40: 95\% CI 1.03-1.91, $\mathrm{p}=0.03$ ), also controlling for age, stage and prior use of chemotherapy.

Conclusions: Cognitive dysfunction is a relevant yet understudied side effect of hormonal therapy in older survivors and may affect the duration of medication adherence. Our preliminary results suggest that survivors with cognitive decline may play a role in early discontinuation of hormonal therapy in older survivors. This will be an important area for future research on temporality of these effects and has clinical implications for survivorship care.

\section{THE NONAGENARIAN PATIENTS WITH CANCER IN} THE UCOG PACA-EST COHORT

C. Rambaud ${ }^{1}$, R. Boulahssass 2 , S. Gonfrier ${ }^{3}$, M. Sanchez ${ }^{2}$,

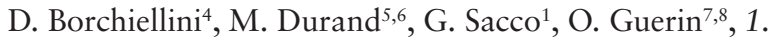

